\title{
La reforma de la policía en Europa: desafíos, debates y alternativas
}

\author{
MANUEL MARTÍN* \\ DiEgo TORRENTE* ${ }^{* *}$
}

\begin{abstract}
Artículo recibido: 30 de agosto de 2015
Artículo aprobado: 15 de abril de 2016

Doi: http://dx.doi.org/10.12804/desafios28.2.2016.03

Para citar este artículo: Martín, M., \& Torrente, D. (2016). La reforma de la policía en Europa: desafíos, debates y alternativas. Desafios, 28(2): 113-144. Doi: http://dx.doi.org/10.12804/ desafios28.2.2016.03
\end{abstract}

\section{Resumen}

La Policía es una institución en continua transformación. Ello responde a la necesidad fundamental de adaptarse a los cambios sociales, económicos y politicos de su entorno. Las organizaciones policiales interpretan el sentido de los mismos y toman decisiones estratégicas que afectan su estructura y funcionamiento. El propósito del presente articulo es analizar la relación entre los cambios recientes en el entorno de las policias europeas y los procesos de reforma de organizativa. La metodología se basa en una revisión de la literatura científica sobre el tema. Se identifican y discuten tres ejes de decisión en torno a las reformas de las organizaciones policiales. El primero tiene que ver con su orientación estratégica global y la elección de un modelo policial de referencia. El segundo se refiere a al grado deseable de apertura de la Policia hacia la colaboración con aliados o partners (públicos, privados y de la sociedad civil) en su búsqueda de respuestas a los retos del entorno. El tercero es transversal; se refiere a la mejor forma en que la policia puede garantizar y maximizar su legitimidad

\footnotetext{
* Docente del Departamento de Sociología de la Universidad de Barcelona. Correo electrónico: diego.torrente@ub.edu

** Profesor e investigador de la Universitat de Barcelona. Correo electrónico: manmartin@ ub.edu
} 
en una sociedad crecientemente fragmentada y desigual. Las tres cuestiones son ineludibles a la hora de considerar cualquier reforma o modernización de la policía boy. El articulo discute las implicaciones de todas ellas y explica las razones por las que la llamada policía de proximidad ofrece una respuesta más satisfactoria tanto a los desafíos sociales, como a los debates académicos. La experiencia europea puede resultar de interés en el contexto latinoamericano.

Palabras clave: reforma policial, cambio organizativo, modelos policiales, partenariado, legitimidad.

\title{
Police Reform in Europe: Challenges, Debates, and Alternatives
}

\begin{abstract}
The police is an institution in permanent transformation. This is because of the need of adaptation to the social, economic and political changes in their environment. Police organizations interpret these changes and make strategic decisions affecting their structure and functioning. The purpose of this paper is to analyze the relationship between recent changes in the European police environment and the processes of organizational reform. The methodology is based on a review of scientific literature on the topic. Three key decisional axes around the reform of police organizations are identified and discussed. The first has to do with the overall strategic model as organization. The second refers to the appropriate degree of openness of police towards collaboration with partners from public, private and civil society sectors. The third one is transversal. It refers to the best way the police can ensure and maximize their legitimacy in an increasingly fragmented and unequal society. The three questions are inescapable when considering any reform or modernization of the police today. The article discusses the implications of all of them and explains the reasons why the so-called "proximity policing" offers a more satisfying answer to both social challenges and academic debates. The European experience may be of interest in the Latin American context.
\end{abstract}

Keywords: Police reform, organizational change, police models, partnership, legitimacy. 


\section{A reforma da Polícia na Europa: Desafios, debates e alternativas}

\section{Resumo}

A polícia é uma instituição em continua transformação. Isso responde à necessidade fundamental de adaptar-se às mudanças sociais, económicas e políticas de seu entorno. As organizações policiais interpretam o sentido dos mesmos e tomam decisões estratégicas que afetam à sua estrutura e funcionamento. O propósito do presente artigo é analisar a relação entre as mudanças recentes no entorno das Polícias europeias e os processos de reforma de organizativa. A metodologia baseia-se em uma revisão da literatura científica sobre o tema. Se identificam e discutem três eixos de decisão em torno às reformas das organizações policiais. O primeiro tem a ver com a sua orientação estratégica global e a eleição de um modelo policial de referência. O segundo se refere ao grau desejável de apertura da Polícia à colaboração com aliados ou partners (públicos, privados e da sociedade civil) na sua busca de respostas aos desafios do entorno. O terceiro é transversal. Refere-se à melhor forma em que a Polícia pode garantir e maximizar a sua legitimidade em uma sociedade crescente fragmentada e desigual. As três questões são inevitáveis à hora de considerar qualquer reforma ou modernização da Polícia hoje. O artigo discute as implicaşões de todas elas e explica as razõos pelas que a chamada Polícia da Proximidade oferece uma resposta mais satisfatória tanto aos desafios sociais, quanto aos debates acadêmicos. A experiência europeia pode resultar de interesse no contexto latino-americano.

Palavras-chave: Reforma policial, mudança organizativa, modelos policiais, partenariado, legitimidade.

\section{Presentación, objetivos y metodología}

La Policía es una institución muy expuesta a su entorno y, por ello, en cambio constante; esta cambia en la medida en que lo hacen los problemas de seguridad, los conflictos sociales, la situación económica y otros factores de su entorno inmediato. Existen evidencias de que, en los últimos años, se han acelerado el ritmo de las reformas que realizan las diferentes Policías europeas (Ellison \& O'Reilly, 2008; Medina, 2011; van Sluis et al., 2013). Se producen cambios tanto a nivel del sistema policial de cada país, como de la estructura y funcionamiento de los cuerpos. A nivel de sistema policial, los cambios van 
en diferentes sentidos. En algunos países, se dan procesos de centralización, mientras en otros se mantienen estructuras descentralizadas. Algunos sistemas tienden hacia modelos más abiertos al mercado, con una presencia amplia del sector privado de la seguridad, mientras que otros mantienen un peso relativo mayor de la seguridad pública. A nivel de las organizaciones, algunas apuestan por la especialización y tecnificación de sus unidades, mientras otras mantienen estructuras generalistas y transversales con fuerte presencia en el territorio. La diversidad de respuestas sugiere que, o bien las realidades nacionales son muy distintas, o bien estas son complejas y se interpretan de forma distinta por los reformadores. La tendencia hacia la integración y convergencia entre los países europeos hace más plausible la segunda opción, pero ello, a su vez, plantea la duda de qué interpretación es más acertada. Resolver esa cuestión implica entender que factores empujan los cambios en la Policía.

Los procesos de reforma de la policía se pueden ver como reacciones a los cambios que ocurren en su entorno. El propósito del presente artículo es tratar de entender la relación entre los cambios recientes en el contexto europeo y las reformas policiales. Esto se hace a partir de los debates académicos sobre el tema. Con ese fin, se realiza un análisis de la literatura científica que relaciona los procesos de reforma de la policía con el contexto donde se producen. Analizando las publicaciones se identifican tres decisiones estratégicas que condicionan el sentido de las reformas; todas ellas surgen como resultado de cierta interpretación de los cambios en el entorno. La primera decisión clave es el tipo de orientación de la actividad policial en su conjunto o modelo policial. Se distingue, por un lado, un modelo político donde el poder político define las prioridades. Existe también, por otro lado, un modelo racional-burocrático o legalista, donde prima la aplicación de la Ley, la especialización y la lucha contra la criminalidad. Finalmente, está el modelo comunitario, en el que la comunidad participa en la definición de los problemas policiales con una orientación hacia la prevención y el mantenimiento del orden. El segundo eje de decisión es el grado de apertura hacia la colaboración con agencias externas y hacia la privatización de la seguridad (Matthews, 1989). El tercer eje es transversal y gira en torno a cómo abordar la cuestión de la legiti- 
midad ante el aumento de la diversidad social y la fragmentación del interés colectivo. $\mathrm{Al}$ mismo tiempo, existen expectativas ciudadanas de mayor eficacia, participación y transparencia, lo que plantea el reto de cómo gestionar las relaciones con la comunidad en un contexto cambiante (Torrente et al., 2015).

El texto que sigue se estructura en cinco partes. En primer lugar, se presenta brevemente el marco teórico que sirve de base para relacionar la reforma de las organizaciones policiales con su entorno. Se comentan algunas de las dimensiones clave del mismo y sus repercusiones sobre la policía. Una vez situado el marco del análisis, se discuten los debates principales en torno a los que giran las reformas policiales. En el segundo apartado, se presenta el debate sobre la orientación estratégica de la policía, o el también llamado "modelo policial". En el tercer apartado, se discute la cuestión de la colaboración, alianza, o partenariados en temas de seguridad de la policía con agencias externas. En el cuarto punto, se discuten las implicaciones de todo lo anterior para la legitimidad de la policía. Finalmente, el artículo se centra en analizar de qué forma el modelo policial de proximidad ofrece una respuesta a las cuestiones que plantean estos debates.

\section{Cambios en el entorno y reforma policial}

En la Sociología de las organizaciones existe una larga tradición de estudios que relaciona los cambios socio-económicos con sus estructuras y funcionamiento. Concretamente, las llamadas teorías de la contingencia ofrecen perspectivas reveladoras. Esos planteamientos surgen en los años sesenta y conciben a las organizaciones como sistemas abiertos en relación al entorno que les rodea (Scott, 1981). El entorno tecnológico (Woodward, 1958), el jurídico, la posición de poder de proveedores y clientes, o la situación en el mercado en relación con la competencia, entre otros elementos, afectan a la estructura y funcionamiento de las organizaciones (Lawrence \& Lorsch, 1967; Thompson, 1967), y a su estilo de liderazgo (Fiedler, 1964). La incertidumbre del entorno es un factor fundamental (Pennings, 1975); por tanto, el entorno es una fuerza que moldea aspectos internos de la organización y, al tiempo, las organizaciones sobreviven 
si adoptan las decisiones adecuadas para adaptarse al mismo. Desde una visión institucionalista, sin embargo, el alcance de los cambios en las organizaciones tiene límites debido a la inercia que suponen la historia, los mitos, las rutinas de trabajo o la capacidad de aprendizaje colectivo. Los párrafos que siguen sitúan la importancia de algunos elementos del entorno de las organizaciones policiales.

Uno de los mecanismos clave por los que los cambios en el entorno de las organizaciones policiales se trasladan a su funcionamiento son las demandas de la población. Estas últimas son dinámicas y varían al ritmo de la sociedad (Martín, 1990). Las demandas que recibe la policía reflejan fielmente la sociedad que las produce. Así, por ejemplo, en las estadísticas de intervenciones policiales españolas del siglo XIX predominan las actuaciones relacionadas con la seguridad física de las personas. Los actos de violencia física, los asesinatos y los riesgos personales, son el motivo más frecuente de demanda ciudadana. Sin embargo, en el siglo XX, los atentados contra la propiedad privada son lo más habitual. Las demandas cambian porque la sociedad es diferente. Hoy día, los peligros físicos han disminuido y la vida humana tiene una mayor protección. La propiedad privada sigue siendo el principal motivo de demanda, pero más centrada en la pequeña delincuencia. Además de ella, el mundo de las drogas, el terrorismo, los delitos relacionados con las nuevas tecnologías, o las redes delictivas internacionales constituyen nuevos vectores de demanda. También lo son la protección del medio ambiente y el medio natural (Torrente, 2001). La población pide dedicación hacia los menores o atención a las víctimas de género; la lucha contra la violencia de género y la protección de los derechos de la mujer absorbe gran número de recursos (Martín 1992 y 1994). No solo cambian las demandas, sino también las visiones y expectativas sobre el rol de la Policía.

La policía tiene que adaptarse a los cambios de forma ineludible. Lo hace a través de la reforma de sus estructuras, estilo de trabajo o de relación con los ciudadanos, entre otros aspectos. Pero la percepción que tiene la Policía sobre el significado de esos cambios no siempre es clara. De hecho, cada vez parece más complejo interpretarlos. Un estudio reciente sobre las Policías españolas muestra que, en los dis- 
cursos sobre el cambio social de los mandos policiales, predominan tres elementos (Torrente et al., 2015). El primero es la percepción de un ciudadano cada vez más diverso y más exigente. El segundo es la diversidad de sus necesidades y demandas. El tercero es su continua evolución y la incertidumbre sobre la dirección de la misma. El alto nivel de exigencia del ciudadano se traduce no solo en unas demandas cada vez más cambiantes y contradictorias, sino en una mayor carga de trabajo. La sensación predominante, como consecuencia de ello, es que sus organizaciones afrontan retos fundamentales y que no está clara la forma mejor para encararlas.

Un elemento crucial del entorno de la policía y a la vez objeto de su trabajo, es la propia sociedad. En el estudio anterior, uno de los cambios sociales que se señalan como de alta repercusión en esta institución es el incremento de la diversidad, tanto social como étnica. La Policía siempre ha tenido mayor dificultad en trabajar en comunidades heterogéneas que en homogéneas. Además, la diversidad cultural plantea cuestiones adicionales. En muchos casos hay fuertes reticencias de algunos colectivos de inmigrantes para acercarse a la Policía. Al tiempo, existen dificultades evidentes de esta para acercarse a ellos. Esas situaciones plantean cuestiones de accesibilidad, pero también de legitimidad. Para alguno de los mandos entrevistados, la dificultad no es solo de falta de comunicación o interacción, sino de distancia entre culturas y valores fundamentales (Torrente et al., 2015).

Algunos estudios señalan que los cambios socio-económicos y tecnológicos se perciben por los propios policías como los que más impactan en las organizaciones policiales. Los cambios políticos y legales les condicionan menos según esa visión (Gascó \& Sallent, 2013). Los cambios sociales en particular (inmigración, cambio de valores, demandas medioambientales etc.) se vivencian de forma negativa y asociados a pérdidas de imagen, legitimidad y autoridad policial; por el contrario, los cambios tecnológicos se vivencian de forma positiva, porque permiten reducir costos, aumentar la eficiencia, facilitar el trabajo y mejorar el contacto con el ciudadano. En relación con los actores, los que se perciben como más importantes son, por este orden, el gobierno, los cuerpos judiciales, la ciudadanía, y otras 
fuerzas de policía. En todos los países se percibe una influencia creciente de los gobiernos, aunque su impacto es moderado (Arjan van den Born et al., 2013).

Los cambios en los problemas de seguridad son otra fuente de la transformación policial. Importa no tanto su volumen, como su naturaleza. Los delitos conectados con los procesos de globalización alteran de forma importante las estructuras y tareas policiales. Existe un incremento en los perfiles de delincuencia más especializada y organizada, y un aumento en la delincuencia internacional, que plantea retos importantes de información e investigación. Afortunadamente, la policía tiene cada vez más acceso a mayor y mejor información; en parte esto es debido a la disponibilidad de grandes bases de datos nacionales e internacionales, cada vez más interconectadas, y de las tecnologías adecuadas para analizarlos. De esa forma, la investigación delictiva gana en profundidad y apoyo en evidencias, pero también se corre el riesgo de desvincularse de la realidad. Ello plantea el debate de hasta qué punto la tecnología puede suplir determinadas tareas policiales y al agente cada día sobre el territorio.

Los cambios tecnológicos son un gran motor de nuevas demandas; la seguridad en las comunicaciones y en Internet supone otro de los grandes retos policiales y una fuente de reformas. Los múltiples delitos que se realizan a través de la web amplían el catálogo policial de la delincuencia. La victimización es alta aunque, en general, las víctimas individuales denuncian poco y menos aún, las corporativas. A pesar de ello, el volumen total de demandas es creciente. No solo es que los nuevos delitos aumentan la carga de trabajo policial, sino que cambian la forma tradicional de trabajar de la policía e impulsan a la especialización técnica, a los nuevos procedimientos y a la innovación organizativa. Pero también están las dificultades que plantea la investigación en la red. Se suman diversos problemas: dificultades de jurisdicción y legales, de encriptación y anonimato, de control de la información por parte de grandes operadores y corporaciones, o de coordinación judicial y policial transnacional. Aparte de estos problemas en su vigilancia e investigación, la red plantea problemas a la policía. Así, en relación al control de manifestaciones, la inme- 
diatez de las convocatorias que realizan los activistas a través de las redes sociales dificulta la capacidad de la policía de anticiparse a los problemas y de controlar las situaciones; además, esta institución también es consumidora activa de. En general, las actitudes hacia ella son positivas, facilitan la gestión de la información, agilizan las respuestas y ayudan a la comunicación con el ciudadano.

\section{Orientación estratégica de la organización}

Una estrategia se refiere a cómo una institución u organización selecciona sus objetivos, orienta sus acciones y establece unas estructuras para conseguirlos (Moore \& Trojanowicz, 1988); las estrategias surgen del diagnóstico de las oportunidades y amenazas del entorno, así como de las fortalezas y limitaciones en sus propias capacidades. Estrategias hay muchas, pero la literatura las ordena en tres grandes modelos estratégicos de policía: el político, el racional-legal, y el comunitario (Ponsaers, 2001; Guillén, 2016). El criterio para ordenarlas es quién tiene más influencia al definir las prioridades de la policía: los políticos, los jueces y el sistema legal, o la población. Además, la literatura policial caracteriza diferentes estilos tácticos y de gestión. Un mismo problema puede abordarse de forma distinta según cuál sea el modelo policial que se sigue. El fenómeno de la inmigración, por ejemplo, está provocando cambios sociales profundos y generando nuevos conflictos en España. Si la policía asume una postura exclusivamente legalista-penal en sus respuestas, realizará principalmente funciones de control y represión. De hecho, una parte de la sociedad y los políticos piden a la policía que adopte ese rol. Si asume una postura comunitaria y de búsqueda del interés general, acabará realizando también labores de atención y protección. Para mantener una postura así, la policía tiene que tener un margen de independencia o, al menos, equidistancia ente la ley, los políticos y la población.

Cada modelo aparece en momentos distintos y responde a entornos diferentes. Tras la Segunda Guerra Mundial, y hasta finales de los setenta, el racional-legal es el dominante en Europa y Estados Unidos (Torrente, 1997), aunque convive con algunas policías más militarizadas y politizadas en España o Portugal (Ballbé, 1983). El entorno 
es de sociedades de bienestar, con una delincuencia común extendida pero abarcable. Sin embargo, el aumento de esta y el descontento de las clases medidas provocan una crisis de eficacia y legitimidad del modelo. El nacimiento de la policía comunitaria en la década de los ochenta pretende responder a esos problemas; este modelo extiende los principios del Problem Solving Policing a las familias, escuelas y asociaciones, haciéndolos socios clave de la policía (Goldstein, 1990) y permitiendo que la comunidad gane capacidad para definir los problemas e incluso los objetivos de algunas policías (Trojanowicz, 1989; Kelling \& Moore, 1988; Greene \& Mastrofski, 1988). La visión de que la policía debe estar cerca de la comunidad incentivó un proceso de descentralización policial en esa década y la siguiente. La adopción del modelo comunitario no resulta fácil debido a que las inercias del modelo reactivo y legalista son importantes (Braga \& Weisburd, 2007; Bayley, 2008). El éxito del modelo es un tema controvertido. Sin embargo, las evidencias acumuladas sostienen que la policía comunitaria funciona bien cuando está focalizada en problemas concretos. Además, está demostrado que mejora los niveles de inseguridad percibida y mejora la legitimidad policial (Weisburd \& Eck, 2004; Skogan, 2006).

Los principios en los que se basan los modelos comunitarios mantienen su influencia, pero, a partir de la década de los noventa, los procesos de globalización hacen aumentar la delincuencia organizada, los delitos tecnológicos y los económicos. Las policías les hacen frente incrementando su especialización, fortaleciendo la colaboración policial internacional o implicando a diversos sectores de la sociedad en la seguridad. Aparecen nuevos desarrollos tácticos que potencian algunos rasgos de los modelos anteriores (Mastrofki \& Willis, 2010). Algunos de ellos pueden considerarse nuevos desarrollos de los principios racional-legales. Así, la tolerancia cero, o Broken Windows Policing, plantea reducir la delincuencia y la inseguridad a base de concentrarse en los problemas de orden en las calles aplicando de forma estricta la ley (Wilson \& Kelling, 1982). El llamado COMPSTAT se basa en generar estadísticas delictivas muy detalladas y geo localizadas, que se utilizan como criterio para adjudicar recursos y evaluar resultados policiales. El llamado Intelligence Led Policing busca potenciar el desarrollo de la inteligencia policial, especialmente en la lucha contra la 
criminalidad organizada y el terrorismo (Jansen \& Bruisma, 1997). El desarrollo de grandes bases de datos y de capacidad de cálculo lo hacen posible (Medina, 2011). El llamado Evidence Based Policing utiliza las evidencias científicas disponibles para orientar las estrategias y la adjudicación de recursos policiales (Sherman, 1998). Este último constituye una táctica más polivalente y aplicable en modelos comunitarios.

El debate del modelo continúa vigente en los procesos de reforma policial actuales, debido a que la sociedad europea realiza demandas muy diversas; por una parte, pide una policía cercana, flexible, de servicios, y transparente y, por otra parte, los problemas de seguridad son cada vez más transnacionales y amenazantes; esto empuja a esta institución hacia la colaboración internacional, la especialización, el manejo de información, el uso de tecnología y la exigencia de referentes legales claros. Aparentemente, existe tensión entre esas dos demandas, porque la primera implica poner al ciudadano en el centro de unas organizaciones polivalentes y flexibles, mientras la segunda implica poner a la legalidad en el centro de organizaciones especializadas y tecnificadas. Los nuevos desarrollos del modelo racionalburocrático centrados en la lucha contra la delincuencia trasnacional implican especialización, grandes unidades de inteligencia o investigación, y gran apoyo judicial. Los nuevos desarrollos del modelo comunitario implican prevención, participación ciudadana, transversalidad y la implicación de la comunidad (Torrente, 1999). Optar por modelos mixtos o híbridos no siempre es fácil porque los principios de funcionamiento son distintos; por tanto, la apuesta por un modelo u otro tiene implicaciones mayores no solo en las organizaciones policiales, sino también en el conjunto de los sistemas nacionales de seguridad. La pregunta entonces es ¿ंson realmente contradictorias estas demandas? ¿Qué modelo garantiza una mejor respuesta a esas demandas tan distintas?

\section{Alianzas y colaboraciones con otras agencias}

Uno de los cambios más notorios de los últimos treinta años es la diversificación de las funciones policiales. Se ha acuñado el término 
pluralization of policing para recoger la idea de que en la provisión de servicios de seguridad participan, de forma creciente, una gran diversidad de agencias y grupos públicos, privados y del tercer sector; así, no solo conviven diferentes actores de la seguridad, sino que se incrementan la colaboración y los partenariados entre ellos. La idea de partenariado consiste en trabajar con otras organizaciones o grupos en un objetivo compartido y en igualdad de poderes. Ese trabajo comprende tanto el diseño como la ejecución de tareas. Todo ello implica también una responsabilidad compartida (Oakley, 2001). Este texto se centra en las alianzas entre la seguridad pública y otros actores.

La policía mantiene una gran diversidad de relaciones con entidades, organizaciones del tercer sector, empresas, grupos de interés, etc. Los contados con ellas son cada vez más regulares. Por ello, las diferentes policías las han institucionalizado creando departamentos de "relaciones con la comunidad", "relaciones ciudadanas" o de "participación ciudadana”. Existen agentes especializados e, incluso, aplicaciones informáticas específicas para gestionar la interacción. Fuera de las organizaciones policiales también aumentan los espacios transversales de relación y cooperación institucional en temas de seguridad. Un ejemplo clásico son las llamadas mesas o consejos de prevención, donde están presentes diversos intereses. Por otra parte, los interlocutores y las formas de interlocución tradicionales de la policía cambian con el tiempo; estos son cada vez más grupos de intereses específicos, no transversales (sindicatos, asociaciones de vecinos, etc.). Ciertos tipos de asociacionismo vecinal, por ejemplo, pierden protagonismo, incluso en municipios y barrios con fuerte tradición. Los movimientos vecinales son más efímeros; se organizan para reivindicaciones concretas y luego se disuelven. Internet es la nueva forma de canalizar las demandas.

Con frecuencia, los policías conciben la sociedad organizada como grupos de presión, pero también como grupos de interlocución. Es decir, como fuentes de información y como forma de control indirecto sobre los afiliados de sindicatos, asociaciones etc. El interés por la relación es mutuo. Los grupos de interés ven una vía de ganar influencia sobre la policía; a través de esos contactos, la información 
se canaliza en los dos sentidos. Por un lado la policía recoge informaciones valiosas y, por otro, sus mensajes llegan a una diversidad de esferas y espacios. Además, dado que se genera confianza mutua, la información que proporcionan se suele percibir por los propios policías como creíble y confiable. En general, la valoración policial de estas colaboraciones es positiva. Incluso, se asocian con la policía comunitaria. Para muchos policías, se trata, en definitiva, de una nueva forma de capital social de la policía.

Entre los académicos también se asume con frecuencia que las alianzas del sector público con otros son siempre positivas porque junta los esfuerzos y experiencia de esas instancias ante problemas complejos (Murphy \& Cherney, 2012). También se da por sentado que las partes trabajan por el bien común (McGuire, 2006). Sin embargo, diversas investigaciones documentan numerosas dificultades. Así, las alianzas con determinados intereses privados originan tensiones por las divergencias en lo que consideran una forma apropiada de intervención (Jacobs, 2010). El grado de las mismas depende del tema de colaboración, la suficiencia de recursos y de si la otra parte ve la seguridad como una responsabilidad suya. El conflicto de roles es un tema central (Worrall \& Gaines, 2006). Otros factores, como las expectativas excesivas o de efectos inmediatos pueden producir decepción. Por otra parte, las organizaciones son una amalgama de intereses y culturas distintos que dificultan las colaboraciones. En ocasiones, como cuando se colabora con asociaciones de minorías étnicas, es esencial generar un clima de confianza previo, pero esto no siempre es fácil (Oakley, 2001).

Uno de los sectores clave para el establecimiento de alianzas es el de la seguridad privada. Existe un debate académico intenso sobre el rol que juega ese sector en la seguridad colectiva; es un debate que, con frecuencia, se cruza con el de la privatización de la seguridad pública (Bosch et al.; 2005, Torrente, 2006). Su importancia reside en que, según como se conciba ese rol, la valoración de las alianzas varía mucho. Existen cuatro grandes visiones. Bajo las teorías liberales, la seguridad privada cubre las limitaciones de la policía para atender las demandadas de seguridad crecientes; esta aparece como complementaria 
a la pública (Cunningham \& Taylor, 1985; Cunningham et al., 1990; Kalakik \& Wilhorn, 1972). Las teorías estructuralistas sostienen que la seguridad privada se adapta mejor que la pública a una sociedad post-industrial individualista, fragmentada y con intereses contradictorios; en este caso, es un reflejo de la tendencia a fragmentar y privatizar los espacios (Shearing \& Stenning, 1987; Matthews, 1989). Las teorías radicales o marxistas argumentan que el sistema capitalista necesita proteger cada vez más sus beneficios de las tensiones y contradicciones sociales que él mismo genera (Spitzer \& Scull, 1977). Finalmente, está el modelo pluralista. Johnston (1992), Jones y Newburn (1998) enmarcan el fenómeno del auge de la seguridad privada en el contexto del desarrollo del sector servicios. Parten de la idea de que la distinción público-privado es reduccionista y carece de fronteras claras. La policía se ve, no tanto como una agencia con una función simbólica, sino como una agencia que vende servicios a un mercado en el que compite con otras agencias públicas, privadas y civiles (Moore \& Trojanowicz, 1988). Jhonston (1992) señala que no existen diferencias intrínsecas entre el mercado y el Estado en la provisión de servicios de seguridad como tampoco existen en términos de salud. De esta manera, ellos sostienen que existe una convergencia creciente entre la seguridad pública y la privada (Jones \& Newburn, 1998). Sin embargo, algunos estudios cuestionan esta asunción, afirmando que los clientes y las demandas son distintas (Boch et al., 2005, Torrente, 2006; Williams, 2005).

Existen varios factores que explican la tendencia hacia el incremento de esas alianzas. La Policía tradicionalmente mantiene contactos con diferentes sectores organizados de la sociedad. Sin embargo, a partir de los años ochenta, la redefinición de la misión policial de "perseguidores de delitos" a "solucionadores de problemas", mediadores, o gestores del miedo favorece la búsqueda de socios y alianzas con ciertos grupos. Otro factor es la necesidad de contar con apoyos en la lucha contra la delincuencia organizada, el terrorismo y otras formas de delincuencia. Originariamente, la estrategia de respuesta a ellas es principalmente penal. Se basa en testimonios de víctimas y una labor de inteligencia criminal. En la medida en que esos problemas evolucionan y se tornan más diversos y complejos, las estrategias se 
diversifican y se buscan alianzas con otros actores públicos, privados y de la sociedad civil (Jansen \& Bruinsma, 1997; Harfield, 2008; Torrente, Valencia \& Bosch 2005). La colaboración con instancias privadas permite también a la policía ganar experiencia en áreas como los delitos de cuello blanco, los fraudes a las compañías de seguros, el fraude financiero, las suplantaciones de identidad, o en seguridad de la información. Todos estos son campos de importancia creciente donde la experiencia del sector privado es muy útil.

La colaboración con sectores organizados de la sociedad civil plantea a la policía diversos beneficios, pero también retos importantes. Quizás uno de los mayores beneficios es que, a través de colaborar, la policía mejora en su capacidad de innovación y adaptación al entorno. Bayley (2008) repasa las principales innovaciones organizativas de la policía en Estados Unidos y concluye que la mayoría han surgido fuera, pero la la institución policial acaba adoptándolas gracias a la colaboración con la sociedad civil (Bayley, 2008). El reto más importante es evitar perder de vista el interés general ante la presión de determinados grupos con intereses concretos y con poder. La búsqueda del interés común es el pilar central de la policía y de su legitimidad. Por suerte, la sociedad es plural y los intereses son muy diversos (Ellison \& O’Reilly, 2008). Sin embargo, el reto sigue estando ahí. Para esta institución no siempre es fácil discernir el interés general; los modelos racional-burocráticos tienen como referente mayor del interés general a la Ley. En los modelos comunitarios, no está tan claro, ni es tan fácil. La pregunta entonces es ¿cómo puede beneficiarse la seguridad pública de las ventajas de la cooperación con otras agencias sin caer en el riesgo de perder de vista el interés general?

\section{La cuestión de la legitimidad}

La legitimidad es un atributo fundamental para las instituciones políticas y las relacionadas con la justicia. Esta depende fundamentalmente de que utilice procedimientos que sean percibidos como justos (Sunshine \& Tyler, 2003). Los elementos formales son centrales en el caso de la justicia, pero en el de la policía, muchas de sus actuaciones no siguen procedimientos formales que faciliten esa percepción. Su legi- 
timidad depende más de que sus actuaciones se valoren como justas y orientadas al bien común. Por ello, los policías tienden a actuar en función de lo que creen que la comunidad considera una respuesta apropiada (Finckneauer, 1976). Contar con un capital de legitimidad es clave para la policía porque facilita su trabajo. Sin embargo, la confianza en ella no depende tanto de los niveles de delincuencia, como de la percepción de estabilidad, cohesión social, y consenso moral del vecindario (Jackson \& Bradford, 2009). La policía se ve como un guardián del orden moral de la comunidad. Por ello, la Policía debe encarnar los valores morales de su comunidad y tratar a las personas con dignidad y de forma justa (Jackson \& Sunshine, 2007).

Tanto la legitimidad, como las actitudes positivas de la población hacia ella, dependen de variables contextuales. En una revisión de más de cien estudios sobre esas actitudes, Ben Brown y William Benedict (2002) señalan que las variables que mejor las explican son la edad, etnicidad, el contacto con la policía, y el grado de (des)organización social del barrio. También las actitudes tienen cierta relación con las que se mantienen hacia el sistema legal en general (Albreicht \& Green, 1977). Por lo tanto, la confianza y la legitimidad dependen de dos factores principales: el primero es mantener el vínculo con la población, y actuar con tacto, especialmente en los barrios difíciles; el segundo es la capacidad de las propias comunidades locales de mantener la cohesión social y el consenso moral. El primer factor está en manos de la Policía; el segundo, solo colateralmente. Ante una sociedad compleja, desigual y fragmentada, la policía experimenta más tensiones y puede tener la tentación de refugiarse en tareas más técnicas o administrativas. Sin embargo, el riesgo de pérdida de confianza y legitimidad es grande. Por otra parte, la percepción de la población sobre la eficacia policial también depende de los contactos personales que ha tenido (Kutnjak, 2008).

Por tanto, de acuerdo a las evidencias, la legitimidad de la policía no se fundamenta en su autoridad legal, su rol de hacer cumplir la ley o en su eficacia anti-delictiva; su legitimidad tiene, sobre todo, una base social y comunitaria. Por supuesto, la cohesión social no depende solo de la Policía. Sin embargo, su dedicación a todo lo que pueda 
afectar la calidad de vida, la convivencia, la seguridad colectiva, o a incrementar la sensación de seguridad fortalece su legitimidad. En ese sentido, identificar y defender el interés colectivo es una condición para la legitimidad de la policía. Sin embargo, la desigualdad, la exclusión y la diversidad social creciente dificultan su búsqueda. Tradicionalmente, la ley ha representado para la policía un elemento clave de dónde reconocer el interés común, pero entre los sectores más desaventajados y excluidos, también existe un cuestionamiento creciente de determinados marcos normativos y políticos. El equilibrio tradicional entre ley, política y población se debilita. La población demanda más participación, más interacción y más transparencia a las instituciones públicas. No es solo que es más complejo gobernar la diversidad, sino que esa diversidad se ha empoderado. En las cuestiones de orden público es donde quizás más se visualizan las tensiones que origina la desigualdad. Así, por ejemplo, la crisis económica del 2008 en España agudiza y hace más visibles algunas de las tensiones ya existentes (Torrente et al., 2015). Llegados a este punto, la pregunta es ¿cómo puede la policía mantener e incrementar su legitimidad y ganarse la confianza ciudadana en un entorno crecientemente desigual, fragmentado, e individualista?

\section{La policía de proximidad como respuesta}

Los apartados precedentes sitúan tres grandes decisiones estratégicas en torno a la reforma de policía en Europa. Cualquier proceso de reforma policial tiene que afrontar esas decisiones: (1) ¿Qué modelo estratégico de policía es capaz de atender mejor la demanda de mayor acercamiento a la población y, a la vez es el más eficaz ante los problemas de seguridad de un mundo globalizado? (2) Asumiendo la magnitud de esos problemas y que la policía no puede afrontarlos por sí sola, ¿cómo tendría que organizarse la colaboración de otras agencias y entidades para no comprometer el interés general que intentan preservar las instituciones de seguridad pública? (3) Sabiendo que la legitimidad de la policía pública es su mayor patrimonio y que esta se apoya principalmente en la existencia de comunidades cohesionadas, ¿cómo puede la policía mantenerla en sociedades crecientemente desiguales, fragmentadas, e individualistas? En este apartado 
argumentamos que, aun no siendo la panacea, los llamados modelos de policía de proximidad son los que pueden ofrecer respuestas más satisfactorias a esas tres preguntas. Para ello, a continuación, se explican con detalle los elementos esenciales del modelo.

El modelo de policía comunitaria es uno de los tres planteamientos estratégicos que nos deja la historia moderna de la policía. Sin embargo, el concepto aparece y se desarrolla en contextos anglosajones, principalmente en Estados Unidos e Inglaterra. En Europa, toma fuerza una adaptación del mismo conocida como policía de proximidad (Guillén, 2016). El término se utiliza de forma amplia en Francia, Italia o Alemania. En España también se utiliza el concepto, no siempre sinónimo, de policía de barrio. La Policía de Proximidad se basa en tres grandes principios. El primero es el compromiso con el tejido social y la cercanía a la población; el segundo es el de prevención como estrategia general para afrontar los problemas, y el tercero es la movilización de distintas agencias públicas, privadas y del tercer sector como aliadas en su labor. A continuación se discuten esos principios y sus implicaciones.

Inicialmente, la idea de fomentar la proximidad entre la policía y la sociedad, como planteamiento de base, es de aceptación general; casi ningún sector de la sociedad defendería hoy que la policía debe mantenerse alejada de la población. Por tanto, la necesidad de proximidad entre la policía y la población coincide con las expectativas ciudadanas mayoritarias. Por otro lado, para esta institución, el acercamiento a la población a la que sirve es un axioma desde su misma creación. Los programas políticos de distintas ideologías o las opiniones en los medios de comunicación, van en una dirección parecida. Nadie cuestiona que la policía tiene que ser un servicio próximo al ciudadano; sin embargo, cuando se trata de situar esas ideas como el principio fundamental de su funcionamiento, el consenso no es tan amplio. Por un lado, la proximidad es un concepto amplio y con distintas interpretaciones; por otro, existen discrepancias al decidir en qué condiciones, o hasta qué punto hay que apostar por la proximidad. En la práctica, sólo un número limitado de responsables políticos y policiales creen que la proximidad tiene que constituir 
el estilo habitual de trabajo de la policía. La mayoría solo lo acepta en determinadas condiciones o solo para algunos temas. Desde una perspectiva interna, con frecuencia, la mayoría de los policías manifiestan su apoyo a la proximidad solo como técnica para mejorar la imagen de la policía o para ampliar la información; no conciben al ciudadano como un socio real. Creen, siguiendo la cultura policial legalista, que el ciudadano tiene la obligación de colaborar con la policía, facilitando información o denunciando delitos. En general, la policía se plantea como una organización a la que estamos obligados a prestar la colaboración ciudadana. Sin embargo, el ciudadano como sujeto pasivo es una concepción superada; buena parte de las resistencias se debe a que el modelo supone un cambio radical respecto a la mentalidad legalista mayoritaria, otras veces, porque no se comprende del todo el modelo.

La filosofía del modelo de proximidad requiere que toda la organización esté orientada hacia la comunidad (principio de cercanía). La policía debe ser capaz de implicarse en el tejido social, conocer a los vecinos y ser conocidos por ellos, detectar problemas potenciales, analizarlos y diseñar medidas adecuadas para que no se produzcan (principio de prevención). El policía se identifica con el ciudadano y asume un rol de analista de la seguridad y de solucionador de problemas. Más importante que reaccionar bien contra el delito, es evitar que este llegue a producirse. Eso solo es posible si conoce en profundidad el terreno, los focos principales de tensión, los términos del conflicto, o los actores clave. Pero también solo es posible si trabaja conjuntamente con distintos sectores sociales de una forma interdisciplinar, yendo a las causas de los problemas y explorando conjuntamente soluciones y alternativas (principio de movilización e implicación de las agencias). Ello implica la creación de canales de comunicación sólidos y estables. La proximidad es, sobre todo, orientar el trabajo policial a la resolución de problemas con una actitud proactiva, preventiva y participativa (Torrente, 1999). Se trata, por tanto, de una manera diferente de entender y hacer policía que representa toda una revolución en la mentalidad, que es necesario extender no solo de los policías, sino también a políticos y jueces. 
Las organizaciones policiales de proximidad desarrollan conceptos distintivos como proximidad, proactividad, prevención, resolución de problemas, mediación, visión global de la situación, planificación de intervenciones, gestión de riesgos, gestión del miedo o atención a la víctima. Se trata de un estilo de gestión de la seguridad que conlleva a una cultura distinta a la tradicional. Valores como el trabajo en equipo, la comunicación con el ciudadano, la orientación a resolver problemas, el trabajo en red con otras policías y agencias son centrales, pero, quizás el más importante es el de implicación con el tejido social. El cambio no es solo organizativo, sino también, individual; cada policía es un auténtico profesional que hace suyos esos valores y es consciente de su propia responsabilidad sobre el territorio o la función asignada. Para ello, ha de disponer de la información suficiente para abordar el problema y de unas estructuras de apoyo y coordinación con el resto de unidades policiales y también con otras agencias (Martin, 1990).

Los modelos de proximidad no son fáciles de aceptar a pesar de que el coste social de la respuesta penal puede llegar a ser inasumible para una sociedad democrática y de derecho. ¿Qué significa que la policía debe ser intolerante con el delito? ¿Hasta dónde hay que llevar ese principio? ¿Son demasiado tolerantes con el delito los policías que trabajan bajo valores de proximidad, mediación, o resolución de problemas? ¿Son rigurosos en la aplicación de la ley únicamente los modelos racional-burocráticos y los de proximidad son anárquicos? ¿Únicamente son los primeros los que defienden la sociedad frente al desorden y la delincuencia mientras que los segundos los toleran? Algo falla en el discurso de la represión. Los resultados obtenidos por las políticas de tolerancia cero, las técnicas de saturación policial en un territorio, o la mera "mano dura" se han demostrado eficaces. Más bien se produce un efecto de desplazamiento de la delincuencia desde una zona a otra con menor presión. Tampoco son fáciles de aceptar los modelos de proximidad para los propios cuerpos policiales dada la inercia de la cultura racional-burocrática. De hecho, la mayoría consideran inapropiado dedicarse a temas relacionados con el entorno, la calidad de vida y otros temas considerados menores y no propiamente policiales. Las referencias a la policía comunitaria o 
de proximidad son frecuentes en la profesión; sin embargo, muchos discursos no la conciben como un verdadero modelo policial, sino que recogen solo algunos aspectos parciales, o prácticos, de la misma. En general, se vincula la Policía de proximidad con la idea de prevención, y con la capacidad de la Policía de obtener información.

\section{Proximidad y problemas globales}

Los apartados precedentes demuestran que el cambio en cualquier organización se inicia cuando el entorno de la misma lo exige. El modelo de proximidad responde a un cambio de fondo en las actitudes y demandas de la población hacia los gobiernos e instituciones de los países desarrollados. Se les exige más participación ciudadana, más interacción, más transparencia, eficacia, y más y mejores servicios. Ciertos sectores de la Administración Pública tradicional aparecen cuestionados por su rigidez organizativa, burocratización y por su concepción del ciudadano como mero receptor pasivo de los servicios. Los sistemas burocráticos weberianos permiten históricamente configurar una Administración Pública altamente garantista, racional, eficaz y orientada por normas; sin embargo, su rigidez, jerarquización y excesiva especialización dificultan el ofrecimiento de respuestas adecuadas a las demandas. En una sociedad moderna, dinámica, diversa y compleja no se acepta que la Administración sea un mundo lejano, burocrático, orientado a crear normas y prohibiciones y de espalda a los problemas reales del día a día.

La policía es parte de la Administración Pública y recibe las mismas demandas. Se le pide una actitud diferente hacia el ciudadano, más interacción, más participación, más transparencia, una renovación en la forma de enfocar de sus tareas, y, en definitiva, un nuevo estilo de hacer Policía. Si en el siglo XIX las intervenciones policiales se relacionan con la integridad física y en el siglo XX con la propiedad privada, en el XXI es la calidad de vida, en su sentido más amplio, el foco principal de las demandas. Atender adecuadamente esa demanda solo puede hacerse desde la cercanía de la Policía con su sociedad. Por otra parte, su legitimidad depende de esa cercanía con el ciudadano. 
Al mismo tiempo, se demanda a la policía que responda de forma eficaz a los nuevos retos globales de la seguridad: delincuencia organizada, terrorismo, delitos tecnológicos, delitos de las organizaciones, corrupción, delitos de las profesiones, etc. Muchas policías responden a esas demandas creando departamentos especializados, incorporando nuevas tecnologías, realizando labores de inteligencia, mejorando su preparación o creando estructuras de cooperación internacional; los dos tipos de demandas son simultáneas y distintas. Por otro lado, se pide una policía muy cercana, generalista, dedicada a los problemas del día a día de la vida en común. Además, se pide una Policía enfocada a los grandes problemas de la seguridad. Ello conlleva una Policía más tecnificada, formada, especializada, hábil en el uso de tecnologías de la información, pero también con mayor peso de las estructuras burocráticas. Los policías evitan presentar estas dos tendencias como contradictoras (Torrente et al., 2015), se presenta la especialización como irremediable, y compatible con la presencia en el territorio y la cercanía al ciudadano. Sin embargo, los recursos son limitados y atender bien ambas demandas no siempre es posible. Pero, más allá de los recursos, está la dificultad de la tensión entre los principios necesarios para realizar una labor de cercanía a las demandas de seguridad cotidianas del ciudadano y las demandas de seguridad del Estado. ¿Suponen esas estructuras una nueva forma de burocratización de la Policía?

Los problemas que atiende la policía de proximidad no son radicalmente distintos de los que atienden esas unidades especializadas. La delincuencia global tiene conexiones locales. Los grandes carteles de narcotraficantes forman una cadena con el pequeño vendedor del barrio, del mismo modo que un delincuente local que roba una cartera necesita estar conectado con una red que le permita vender las tarjetas de crédito. Lo mismo ocurre con el fraude alimentario, el tráfico de animales protegidos o el vertido clandestino de desperdicios tóxicos. La policía tiene que dar respuesta a la delincuencia de alta especialización y, a su vez, a la pequeña delincuencia cotidiana. Separar ambos niveles resta eficacia al sistema de seguridad. Despreciar la importancia del contacto diario sobre el territorio y fiarlo todo a las bases de datos o a la tecnología es un error. Así lo demuestra el 
fracaso de la CIA, con su enorme infraestructura tecnológica, para prevenir los ataques terroristas del 11S. Lo contrario también es un error. La confluencia de esas dos estrategias fortalece al sistema de seguridad. La dificultad principal es que ambas responden a principios y filosofías de trabajo distintas.

El funcionamiento de muchas de esas nuevas estructuras de lucha contra la delincuencia global tiene muchos rasgos racional-burocráticos; la burocracia potencia la especialización y el uso de la tecnología. En la cultura organizativa, un agente adscrito a una unidad especializada es, aparentemente, un policía más profesional. Internamente, suele estar mejor considerado, que el patrullero generalista. A menudo, tanto las funciones que se le asignan, como las herramientas puestas a su disposición, determinan más su prestigio profesional que los resultados obtenidos. Ello crea una tendencia cultural en las organizaciones policiales racional-burocráticas hacia la diferenciación entre policías, unidades, departamentos y cuerpos. Se crean, en muchos casos, auténticos reinos de taifas. De esa forma, se llega a dedicar más energía a defender el espacio corporativo que a resolver los problemas reales de la comunidad. La diversificación y especialización llega muchas veces al absurdo. Aparecen policías que trabajan de espaldas unos a otros, de forma descoordinada (cuando no opuesta), solapándose en el territorio, en los temas o con los mismos ciudadanos. Se dan casos de patrulleros que, por ir en moto, a pie o en coche, se ignoran mutuamente. Existen policías uniformados y policías sin uniforme viviendo en mundos aislados. Son algunos de los problemas clásicos de la burocracia que tanta contestación social reciben.

El modelo de proximidad rompe con esta filosofía diferenciadora de la burocracia. La proximidad no es una especialidad más. No se trata de unos cuantos agentes dedicados a hablar amablemente con los vecinos o dedicándose a tareas asistenciales, mientras el resto de compañeros se dedican a hacer de policía 'de verdad'. La proximidad es una filosofía, un estilo de trabajo que afecta a todo el cuerpo policial, no solo a unos cuantos. La policía trabaja de manera interdisciplinar, integral y profesional para prevenir y resolver problemas concretos de seguridad y calidad de vida de la comunidad. Ello no es incompatible 
con el uso de la tecnología, el manejo de bases de datos, o la creación de estructuras de inteligencia, eso sí, su uso será más preventivo que reactivo. Para ello es necesario que toda la policía se configure como un sistema cohesionado donde todos los agentes, con independencia de la unidad a la que estén adscritos, trabajan de forma coordinada. La eficacia es mayor cuando trabajan juntos patrulleros, analistas de información, investigadores, agentes a pie y motorizados, uniformados y no uniformados. Por otra parte, su cultura de alianzas con otras agencias facilita la cooperación para prevenir atentados u otros problemas. A pesar de ello, siempre la cooperación de cuerpos policiales diferentes es más fácil cuando existe un sistema de autoridad y de reparto de competencias bien definido. Cuando esto no es así, o existe ambigüedad, la percepción de los cuerpos es que solo pueden contar en la práctica con sus propios recursos. En la medida en que las organizaciones adopten la filosofía de la proximidad se van haciendo más flexibles, dinámicas y capaces de adaptarse a cualquier demanda del entorno, incluyendo la lucha contra las formas de delincuencia más internacional.

\section{Interés general y cooperación público-privada}

La cooperación de la policía con otras agencias y organizaciones es una práctica extendida, esta forma parte de los rasgos definitorios del modelo comunitario; sin embargo, se ha generalizado entre los cuerpos racional-burocráticos o legalistas. El debate principal que se suscita es hasta qué punto existe el riesgo de renunciar al interés general en base ciertas ventajas de la cooperación. Antes de responder a esa cuestión, hay que plantear una previa: ¿Hasta qué punto esa cooperación es necesaria para la seguridad colectiva? Se comentan a continuación varios casos que ayudan a responder a la cuestión.

Una de las tareas más comunes que realiza la Policía es dar respuestas a los conflictos de convivencia entre ciudadanos de cualquier condición. Muchos de ellos se enmarcan en conflictos sociales de fondo, lo que los convierte en una cuestión difícil e incómoda para los policías. Gestionarlos todos y llegar al conflicto cero es una utopía (de Miguel \& Martín, 1995). La Policía se enfrenta de forma permanente a ten- 
siones entre diferentes colectivos. Lo hace, a menudo, en entornos muy desestructurados, y soportando la presión de la sociedad para que dé respuestas a los problemas que ocasiona la mala convivencia. La expectativa es exagerada e irrealista y, por ello, le conlleva una gran tensión. La policía solo puede empezar a ofrecer respuestas adecuadas cuando actúa en coordinación con otras agencias sociales. No es razonable esperar que solo con el esfuerzo de la policía baste. Es necesario mantener canales de comunicación con otros departamentos de la Administración con competencias en el tema. Todo esto, no obstante, no es suficiente. Esos conflictos cambian con el tiempo, lo que hace necesario una formación continuada de los policías y una renovación de las entidades de colaboración.

En ocasiones se atribuye a la Policía la responsabilidad por la degradación del entorno urbano, sin que cuente con las herramientas necesarias para su gestión, ya que no cuenta con los recursos, preparación o competencias para establecer programas de prevención. Responsabilizar a la policía de todo tipo de problemas sociales lleva a un sentimiento de frustración en los propios agentes. Con frecuencia, se les exige una acción meramente reactiva y represiva que, en la práctica, no soluciona el problema, al contrario, sus actuaciones, al estar mal enfocadas, acaban convirtiéndose en un problema para la convivencia y en fuente de desorden e inseguridad. Pretender hacerles responsables únicos es irrealista. Al mismo tiempo, los departamentos de la Administración Pública responsables de esos problemas, y que disponen de las competencias y los recursos correspondientes para ello, se ven frecuentemente libres de la presión pública y de la responsabilidad en la degradación de dicho entorno.

De la misma forma, hoy no es posible afrontar problemas complejos como la inmigración, drogas, circulación u otros similares desde una perspectiva únicamente policial. La policía forma parte de un sistema social mucho más complejo. Existen muchas agencias y profesionales implicados en cada uno de esos problemas. El policía no es un asistente social, ni un abogado, ni un psicólogo; sin embargo, debe actuar de forma interdisciplinar con estos y otros profesionales. Llevando la cuestión más lejos, tampoco puede prescindir de la ayuda de 
la población. La policía de proximidad precisa de recursos humanos, materiales, tecnológicos, económicos y normativos (Martín, 2001), pero también necesita sentido común, información fiable y capacidad para analizar la realidad. Para ello, el principal recurso de la Policía es la población. Trabajar codo con codo con el resto de agentes sociales o con los ciudadanos le permite no solo obtener información, sino también analizarla, conocer los factores relevantes y, con frecuencia, llegar a las causas de los problemas. Aun considerando las limitaciones de los partenariados que señalan los estudios mencionados, todos estos ejemplos hacen pensar que no es posible renunciar a esas alianzas sin un coste importante en términos de eficacia policial.

Las ventajas de la cooperación se pueden convertir en riesgos si no se garantiza que el interés colectivo de la seguridad prevalece sobre el de los grupos asociados. Desde la perspectiva de los propios policías, esas alianzas se valoran positivamente y no se perciben como una forma de "privatización" de la seguridad colectiva (Torrente et al., 2015). Sin embargo, a pesar de que, en la práctica la policía establece alianzas con una gran pluralidad de actores e intereses, ese riesgo existe. La única manera de evitarlo es ejercer el liderazgo de la seguridad pública. Es decir, que la policía identifique y defina previamente dónde está el interés colectivo en un tema determinado. Por supuesto, en las negociaciones los diferentes grupos intentan poner su interés por delante. La habilidad de la policía consiste en llegar a compromisos que no comprometan el interés general y, al tiempo, consigan involucrarlos. Para negociar bien, es necesario conocer a los distintos interlocutores, sus intereses y objetivos, así como las posibilidades y los límites de la colaboración con ellos.

\section{Legitimidad y fragmentación social}

La legitimidad de la policía pública se basa en su contribución a mantener la cohesión en sus comunidades. La cercanía a su día a día y la defensa del interés colectivo son dos aspectos irrenunciables para conservarla, pero la fragmentación y las desigualdades crecientes de las ciudades dificultan ambas cuestiones. A pesar de las dificultades, no hay otra alternativa. La policía de proximidad se orienta hacia la 
mejora de la cohesión social y ofrece algunos recursos para ese fin. Cuanto más fragmentada está una sociedad, más necesaria es su labor. En contextos de gran diversidad y desigualdad, la policía no puede limitarse a esperar que se produzca el conflicto para reaccionar, pues, de ser así, los conflictos acaban por desbordarla y corre el riesgo de perder el control. Es necesario anticiparse al conflicto, diagnosticarlo y gestionarlo, lo que implica un conocimiento detallado del entorno social y el trabajo conjunto con la población; es necesario ir más a las causas que a los efectos.

El conflicto social y la desigualdad se manifiestan en el día a día de las calles. El mantenimiento de un espacio público seguro es un objetivo de cualquier policía con independencia de su modelo organizativo. Sin embargo, muchas veces acaba convertida en un guardián del orden y la moral frente a una sociedad reticente a abordar seriamente los conflictos sociales y que pide a la policía que 'meta la basura debajo de la alfombra', fuera de la vista. Es lo que suele ocurrir en actuaciones con indigentes sin techo, vendedores ambulantes sin licencia, menores díscolos, desocupados, colectivos marginales y otras personas 'potencialmente peligrosas'. En muchos casos, los modelos legalistas realizan actuaciones orientadas normativamente, y hechas con unos recursos limitados, que acaban siendo cuestionadas por abuso de autoridad, malos tratos o falta de sensibilidad social. La policía de proximidad, por el contrario, intenta involucrar a distintas instituciones y asociaciones en la labor. Su capacidad de implicación en el tejido social, junto con la de mediación en los conflictos, hacen de esta un instrumento eficaz para la convivencia de culturas, razas y sensibilidades diferentes. Todo ello contribuye a afianzar su legitimidad.

Resumiendo, este artículo se interesa por los procesos de reforma policial. En Europa existe una larga experiencia en ese terreno y también una tradición académica de investigación. El repaso de esa literatura identifica tres decisiones clave a la hora de acometer reformas que permitan a la policía adaptarse a su entorno. El dilema central es la apuesta por un modelo estratégico. Después está la duda de las ventajas e inconvenientes de establecer alianzas estratégicas con actores externos. Finalmente, y relacionada con esas dos, está la cuestión de 
cómo mejorar la legitimidad de la Policía en contextos de gran diversidad y desigualdad social. El artículo analiza esos debates y, con base en las evidencias que aporta, intenta dar una respuesta a los mismos. Se argumenta que, a pesar de no ser la panacea, los modelos de policía de proximidad, que apuestan por la colaboración con otras agencias, son los que ofrecen respuestas más satisfactorias.

Reformar organizaciones policiales para concebirlas como sistemas de proximidad orientados a la resolución de problemas sigue siendo un gran reto. Sin embargo, dadas las evidencias que tenemos, la proximidad es mucho más que una opción entre otras. La alternativa es mantener policías burocratizadas, que difícilmente sintonizan con las demandas de la sociedad actual. Según Reiss (1971), al planear la policía del futuro, se debe intentar prever los cambios sociales que la afectarán y pensar cómo debería organizarse para afrontarlos mejor. Siempre es necesaria una lectura permanente de la realidad social (Reiss, 1971). La reforma de la policía hay que entenderla como un proceso ecológico de adaptación de las organizaciones a su medio social, un medio que se torna progresivamente más complejo, desigual, contradictorio y paradójico. Sin embargo, la única alternativa para entender e interpretar bien esos cambios y responder adecuadamente es prestar atención a las necesidades y demandas de la población.

\section{Referencias}

Albreicht, S. L. \& Green, M. (1977). Attitudes toward the Police and the Larger Attitude Complex. Implications for Police-Community Relationships. Criminology 15(1), 67-86.

Ballbé, M. (1983). Orden público y militarismo en la España Constitucional (18121983). Madrid: Alianza Editorial.

Bayley, D. H. (2008). Police reform: Who done it? Policing and Society 8(1), 7-17.

Bosch, J. L, Farrás, J., Martín, M., Sabaté, J. \& Torrente, D. (2005). Estado, mercado y seguridad ciudadana: Análisis de la articulación entre la seguridad pública y privada en España. Revista Internacional de Sociología 39, 107-137. 
Braga, A. A. \& Weisburd D. L. (2007). Police Innovation and Crime Prevention: Lessons Learned from Police Research over the Past 20 Years. National Institute of Justice (NIJ).

Brown, B. \& Benedict, W. R. (2002). Perceptions of the police: Past findings, methodological issues, conceptual issues and policy implications. Policing and Police Models. Policing: An International Journal of Police Strategies \&o Management 25(3), 543-580.

Cunningham, W., Strauchs, J. J. \& Van Meter, C. W. (1990). The Hallcrest Report II: Private Security Trends 1970-2000. Boston: Butterworth-Heinemann.

Cunningham, W. \& Taylor, T. H. (1985): Private Security and Police in America: The Hallcrest Report. Porland: Chancellor Press.

De Miguel, J. M., \& Martín, M. (1995). Matar al padre: Un modelo de reconstrucción del conflicto social desde la Sociología de las Organizaciones. Papers: Revista de Sociología 47, 31-53.

Ellison, G., \& O’ Reilly C. (2008). Ulster's policing goes global: The police reform process in Northern Ireland and the creation of a global brand. Crime, Law and Social Change 50, 331-351.

Fiedler, F. E. (1964). A Contingency Model of Leadership Effectiveness. En L. Berkowitz (Ed.), Advances in experimental social psychology (pp. 149-190). New York: Academic Press.

Finckenauer, J. O. (1976). Some factors in police discretion and decision making. Journal of Criminal Justice 4, 29-46.

Gascó, M. \& Sallent, E. (2013). El Proyecto COMPOSITE sobre gestión del cambio en las organizaciones policiales. Revista Catalana de Seguretat Pública 26, 123-134.

Goldstein, H. (1990). Problem Oriented Policing. Nueva York: McGraw-Hill. Greene, J., \& Mastrofski, S. (Eds.) (1988). Community Policing: Rhetoric or Reality? Nueva York: Praeger.

Guillen, F. (2016). Modelos de Policía. Hacia un modelo de seguridad plural. Barcelona: Bosch Editor.

Harfield, C. (2008). The organization of 'organized crime policing' and its international context. Criminology \& Criminal Justice 8(4), 483-507.

Woodward, J. (1958). Management and Technology. London: Her Majesty's Stationery Office.

Jackson, J. \& Bradford, B. (2009). Crime, policing and social order: on the expressive nature of public confidence in policing. The British Journal of Sociology 60(3), 493-521. 
Jackson, J. \& Sunshine, J. (2007). Public Confidence in Policing: A NeoDurkheimian Perspective. British Journal of Criminology 47, 214-233.

Jacobs, K. (2010). The Politics of Partnerships: A Study of Police and Housing collaboration to Tackle anti-social Behavior on Australian Public Housing Estates. Public Administration 88(4), 928-942.

Jansen, F. E., \& Bruinsma, G. J.N. (1997). Policing organized crime: A new direction. European Journal on Criminal Policy and Research 5(4), 85-98.

Johnston, L. (1992). The Rebirth of Private Policing. Londres: Routledge.

Jones, T. \& Newburn, T. (1998). Private Security and Public Policing. Nueva York: Oxford University Press.

Kakalik, J. S. \& Wildhorn, S. (1972). Private Police in the United States (The Rand report). Washington: National Institute of Law Enforcement and Criminal Justice.

Kelling, G. \& Moore, M. (1988). From Political to Reform to Community: The Evolving Strategy of Police. En J. Greene \& S. Mastrofski (Eds.), Community Policing: Rhetoric or Reality? (pp. 1-26) Nueva York: Praeger.

Kutnjak, S., (2008). A Comparative Study of Public Support for the Police. International Criminal Justice Review 18(4), 406-434.

Lawrence, P. R., \& Lorsch, J. V. (1967). Organization and Environment: Managing Differentiation and Integration. Cambridge: Harvard Business School Press. Martín, M. (1990). La profesión de policía. Madrid: CIS-Siglo XXI.

Martín, M. (julio-septiembre, 1992). Policía, profesión y organización: hacia un modelo integral de la Policía en España. Revista Española de Investigaciones Sociológicas 59, 205-222.

Martín, M. (1994), Mujeres policías. Madrid: CIS-Siglo XXI.

Martín, M. (2001), La financiación de las Policías Locales. Cuadernos de la Guardia Civil: Revista de seguridad pública 23, 75-84.

Mastrofski, S. D. \& Willis, J. J. (2010). Police Organization Continuity and Change: Into the Twenty-first Century. Crime and Justice 39(1), 55-144.

Matthews, R. (1989). Privatizing Criminal Justice. Londres: Sage.

McGuire, M. (2006). Collaborative Public Management: Assessing what we know and how we know it. Public Administration Review, 66(1), 33-43.

Medina, J. (2011). Politicas y estrategias de prevención del delito y seguridad ciudadana. Madrid: Edisofer.

Moore, M. H. \& Trojanowicz, R. C. (1988). Corporate Strategies for Policing. Cambridge: National Institute of Justice, U.S. Department of Justice. 
Murphy, K., \& Cherney, A. (2012). Understanding cooperation with Police in a diverse society. British Journal of Criminology, 52,181-201.

Oakley, R. (2001). Building Police-Community Partnerships: UK and European Experience. Australian Institute of Criminology.

Pennings, J. M. (1975). The Relevance of the Structural-Contingency Model for Organizational Effectiveness. Administrative Science Quarterly 20(3), 393-410.

Ponsaers, P. (2001). Reading about "Community (Oriented) Policing" and Police Models. Policing: An International Journal of Police Strategies \& Management, 24(4), 470-496.

Reiss, A. J. (1971). The Police and the Public. New Haven: Yale University Press. Scott, W. R. (1981). Organizations: Rational, Natural, and Open Systems. Englewood Cliffs NJ: Prentice Hall Inc.

Shearing, C. D. \& Stenning, P. (Eds.). (1987). Private Policing Newbury Park: Sage.

Sherman, L. (1998). Evidence-Based Policing, Ideas in American Policing. Washington: Police Foundation.

Skogan, W. (2006). The Promise of Community Policing. En David L. Weisburd \& Braga, A. A. Police Innovation: Contrasting Perspectives. New York: Cambridge University Press.

Spitzer, S. \& Scull, A. Scull. (1977). Privatization and Capitalist development: the case of Private Police. Social Problems 1(25), 18-29.

Sunshine, J. \& Tyler, T. R. (2003). The Role of Procedural Justice and Legitimacy in Shaping Public Support for Policing. Law \& Society Review 37(3), 513-548.

Thompson, J. D. (1967). Organizations in Action: Social Science bases of Administrative Theory. McGraw-Hill: New York.

Torrente, D. (1997). La sociedad policial: Poder, trabajo y cultura en una organización local de Policía. Madrid. Barcelona: CIS y Universidad de Barcelona.

Torrente, D. (1999). Prevención del delito y futuro de la Policía. Revista Española de Investigaciones Sociológicas, 85, 85-94.

Torrente, D. (2001). Desviación y delito. Madrid: Alianza Universidad.

Torrente, D. (2006). Vendiendo seguridad: servicios, conflictos y estrategias de la seguridad privada en España. Sistema. Revista de ciencias sociales 192, 77-104. 
Torrente D., Valencia V. \& Bosch, J. L. C. (2005). Organizando la seguridad: análisis organizativo de los servicios privados de seguridad en España. Politica y sociedad 42(3), 185-208.

Torrente, D., Jaime, O., Sabater, J., Folguera, J. \& Peñaranda, V. (2015). Cambio en las demandas de seguridad y políticas policiales en España. XII Congreso de la AECPA (San Sebastián).

Trojanowicz, R. (1989). Preventing Civil Disturbances: A Community Policing Approach. East Lasing: Michigan State University.

Van den Born, A. et al. (2013). Policing opportunities and threats in Europe. Journal of Organizational Change Management, 26(5), 811-829.

Van Sluis, A., Cachet, L., Jochoms, T., Ringeling, A. \& Sey, A. (Eds.). (2013). Contested Police Systems: Changes in the Police Systems of Belgium, Denmark, England and Wales, Germany and the Netherlands: Eleven Publising.

Weisburd, D. \& Eck, J. (2004). What Can Police Do to Reduce Crime, Disorder, and Fear? Annals of the American Academy of Political and Social Science, 593, 42-65.

Williams, J. W. (2005) Reflections on the Private versus Public Policing of Economic Crime. Bristish Journal of Criminology 45, 316-339.

Wilson, J. Q. \& Kelling, G. L. (1982). Broken windows. The Police and Neighborhood Safety. The Atlantic Monthly 249(3), 29-38.

Worrall, J. L. \& Gaines, L. K. (2006). The effect of police-probation partnerships on juvenile arrests. Journal of Criminal Justice 34, 579-589. 